\title{
POSITIVE TEMPERATURES ON A SEMI-INFINITE ROD $\left({ }^{(}\right)$
}

\author{
BY \\ D. V. WIDDER
}

1. Introduction. In these Transactions the author $[1944 ; 85]\left({ }^{2}\right)$ showed that any solution $u(x, t)$ of the heat equation

$$
\frac{\partial^{2} u}{\partial x^{2}}=\frac{\partial u}{\partial t}
$$

which is non-negative for positive time and zero initially, $u(x, 0+)=0$, is identically zero. It was also proved that any solution non-negative for $t>0$ must have the form

$$
u(x, t)=(4 \pi t)^{-1 / 2} \int_{-\infty}^{\infty} e^{-(x-y)^{2} / 4 t} d \alpha(y),
$$

where $\alpha(y)$ is nondecreasing. Later P. Hartman and A. Wintner [1950; 367] obtained analogous results for temperatures of a finite rod $(a<x<b)$. It is the purpose of the present paper to investigate the corresponding questions for the half-infinite rod $(0<x<\infty)$.

There are two classical problems for the semi-infinite rod which may be phrased as follows:

A. Determine the subsequent temperatures of the rod if it is initially at temperature $f(x)$ and if the finite end is held at temperature $0 ; u(x, 0)=f(x), u(0, t)$ $=0$.

B. Determine the subsequent temperatures if it is initially at temperature 0 and if the finite end is maintained at a variable temperature $\phi(t) ; u(x, 0)=0$, $u(0, t)=\phi(t)$.

Under suitable conditions on the boundary functions the solutions are known to be (compare H. S. Carslaw and J. C. Jaeger $[1948 ; 40-44]$ ):
A. $\quad u(x, t)=(4 \pi t)^{-1 / 2} \int_{0}^{\infty}\left[e^{-(x-y)^{2} / 4 t}-e^{-(x+y)^{2} / 4 t}\right] f(y) d y$.
B. $u(x, t)=(4 \pi)^{-1 / 2} x \int_{0}^{t}(t-y)^{-3 / 2} e^{-x^{2} / 4(t-y)} \phi(y) d y$.

Presented to the Society, December 29, 1952; received by the editors October 18, 1952.

(1) Work supported by the Office of Naval Research, Project N5ori-07634, at Harvard University.

(2) Numbers in brackets refer to the bibliography at the end, the first to the date of publication, the second to the page. 
Both these integral transforms carry positive functions into positive functions in the region of the variables under discussion. It is natural to inquire whether the totality of all positive temperature functions can be obtained in this way. The answer is essentially affirmative. One has only to replace the above transforms of positive functions by Stieltjes integral transforms of increasing functions. The two principal results of the paper follow:

I. Any solution of (1.1) which is $\geqq 0$ for $x>0, t>0$ and which is zero on the $x$ - and $t$-axes is identically zero.

II. Any solution of (1.1) which is $\geqq 0$ for $x>0, t>0$ must equal

$$
\begin{aligned}
& (4 \pi t)^{-1 / 2} \int_{0}^{\infty}\left[e^{-(x-y)^{2} / 4 t}-e^{-(x+y)^{2} / 4 t}\right] d \alpha(y) \\
& +(4 \pi)^{-1 / 2} x \int_{0}^{t}(t-y)^{-3 / 2} e^{-x^{2} / 4(t-y)} d \beta(y),
\end{aligned}
$$

where $\alpha(y)$ and $\beta(y)$ are nondecreasing.

In connection with the first result one must make precise what is meant by the vanishing of a function on the axes, as the example

$$
u(x, t)=x t^{-3 / 2} e^{-x^{2} / 4 t}
$$

shows. It is positive in the quadrant in question and $u(x, 0+)=u(0+, t)=0$ for all positive $x$ and $t$; yet it is not identically zero. Our interpretation of the boundary condition is that $u(x, t) \rightarrow 0$ as $(x, t) \rightarrow$ a boundary point in the twodimensional way. The above function fails to approach zero as $(x, t) \rightarrow(0,0)$.

2. Tychonoff's theorem. We shall need a uniqueness theorem of $\mathrm{A}$. Tychonoff [1935; 199]. A proof of this result was given by the author $[1944 ; 88]$, but for the reader's convenience a statement of the theorem will be given here.

We say that $u(x, t)$ belongs to class $H, u(x, t) \in H$, in a domain if it of class $C^{2}$ and satisfies the heat equation

$$
\frac{\partial^{2} u}{\partial x^{2}}=\frac{\partial u}{\partial t}
$$

there; $u(x, t) \in H$ in any region (which may be closed) if that region can be enclosed in a domain where $u(x, t) \in H$.

We now state the Tychonoff result in two parts.

THEOREM A. If

1. $u(x, t) \in H, a<x<b, 0<t \leqq c$,

2. $\lim$ inf $u(x, t) \geqq 0$ as $(x, t) \rightarrow\left(x_{0}, t_{0}\right)$ when $x_{0}=a$ or $b, 0 \leqq t_{0} \leqq c ;$ or $t_{0}=0$ $a \leqq x_{0} \leqq b$,

then

$$
u(x, t) \geqq 0, \quad a<x<b, 0<t \leqq c .
$$


Theorem B. If

1. $u(x, t) \in H$ in the strip $0<t \leqq c$,

2. lim inf $u(x, t)=0$ as $(x, t) \rightarrow\left(x_{0}, 0+\right),-\infty<x_{0}<\infty$,

3. $f(x)=\operatorname{Max}_{0<t \leq c}|u(x, t)|$,

4. $f(x)=O\left(e^{a x^{2}}\right), x \rightarrow \pm \infty$, for some constant $a$, then $u(x, t)=0$ throughout the strip $0<t \leqq c$.

We conclude this section with the following notation for the "source solution" of the heat equation (2.1):

$$
k(x, t)=(4 \pi t)^{-1 / 2} e^{-x^{2} / 4 t},
$$$$
-\infty<x<\infty, 0<t<\infty .
$$

3. A uniqueness theorem for a half-strip. We begin by a modification of Theorem B that will apply to half the strip $(x>0)$ employed therein.

THEOREM 1. If

1. $u(x, t) \in H, 0<x<\infty, 0<t \leqq c$,

2. $\lim u(x, t)=0$ as $(x, t) \rightarrow\left(x_{0}, 0+\right), 0 \leqq x_{0}<\infty$,

3. $\lim u(x, t)=0$ as $(x, t) \rightarrow\left(0+, t_{0}\right), 0 \leqq t_{0} \leqq c$,

4. $f(x)$ is defined as in Theorem $\mathrm{B}$,

5. $f(x)=O\left(e^{a x^{2}}\right), x \rightarrow+\infty$, for some constant $a$, then $u(x, t)=0$ for $0<x<\infty, 0<t \leqq c$.

We emphasize that in Hypotheses 2 and 3 the approach is two-dimensional (rather than along a normal to the boundary). Consider, for example, the function (1.2) which satisfies $1,4,5$, and for which $u(0+, t)=0,0<t \leqq c$, $u(x, 0+)=0,0<x<\infty$. But this function has no two-dimensional limit as $(x, t) \rightarrow(0,0)$.

To prove the theorem set, for $R>0$,

$$
U_{R}(x, t)=f(R) k(x-R, t) .
$$

Then

$$
|u(R, t)| \leqq(4 \pi c)^{1 / 2} U_{R}(R, t), \quad 0<t \leqq c .
$$

Hence on the segment $x=R, 0<t \leqq c$ we have

$$
(4 \pi c)^{1 / 2} U_{R}(x, t) \pm u(x, t) \geqq 0 .
$$

Also

$$
\lim \inf \left[(4 \pi c)^{1 / 2} U_{R}(x, t) \pm u(x, t)\right] \geqq \lim \pm u(x, t)=0
$$

as $(x, t) \rightarrow\left(x_{0}, 0+\right), 0 \leqq x_{0} \leqq R$, by Hypothesis 2 . The same inequality holds as $(x, t) \rightarrow\left(0+, t_{0}\right), 0 \leqq t_{0} \leqq c$, by Hypothesis 3 . In fact, we could replace "lim inf" by "lim" except at the one point $(R, 0)$. We are now in a position to apply Theorem A to the two functions (3.1) and conclude that

$$
|u(x, t)| \leqq(4 \pi c)^{1 / 2} U_{R}(x, t)
$$


throughout the rectangle $0<x<R, 0<t \leqq c$. Now keep $(x, t)$ fixed in that rectangle and let $R \rightarrow \infty$. By Hypothesis 5

$$
U_{R}(x, t)=O\left(e^{a R^{2}} e^{-R^{2} / 4 t}\right), \quad R \rightarrow \infty .
$$

Hence $u(x, t)=0$ if $0<t<1 /(4 a)$. If $c \leqq 1 /(4 a)$, the proof is complete. Otherwise, repeat the above argument with $u(x, t)$ replaced by $u\left(x, t+(4 a)^{-1}\right)$, and so forth.

4. Properties of certain integral transforms. Let us introduce a condition upon a function $\phi(y)$ sufficient to guarantee that the integral transform

$$
F(x, t)=\int_{0}^{\infty}[k(x-y, t)-k(x+y, t)] \phi(y) d y
$$

shall define a function $F(x, t)$ which belongs to $H$.

THEOREM 2. If

1. $\int_{0}^{\infty} e^{-a y^{2}}|\phi(y)| d y<\infty$ for some $a>0$,

2. $F(x, t)$ is defined by the integral (4.1),

then $F(x, t) \in H$ in $0<x<\infty, 0<t<1 /(4 a)$.

For,

$$
F(x, t)=\int_{0}^{\infty} k(x-y, t) \phi(y) d y-\int_{-\infty}^{0} k(x-y, t) \phi(-y) d y .
$$

Each of these integrals is a special case of one treated in Theorem 1 of the author's paper $[1944 ; 88]$, so that the conclusion is immediate.

TheOREM 3. If in addition to the hypotheses of Theorem 2, $\phi(0+), \phi\left(x_{0}+\right)$ and $\phi\left(x_{0}-\right)$ exist, $x_{0}>0$, then
A. $\lim \sup |F(x, t)| \leqq \operatorname{Max}\left(\left|\phi\left(x_{0}+\right)\right|,\left|\phi\left(x_{0}-\right)\right|\right)$ as $(x, t) \rightarrow\left(x_{0}, 0+\right)$,
B. $\lim \sup |F(x, t)| \leqq|\phi(0+)|$ as $(x, t) \rightarrow(0+, 0+)$,
C. $\lim F(x, t)=\phi\left(x_{0}+\right)$ when $\phi\left(x_{0}+\right)=\phi\left(x_{0}-\right)$, as $(x, t) \rightarrow\left(x_{0}, 0+\right)$,
D. $\lim F(x, t)=0$ as $(x, t) \rightarrow\left(0+, t_{0}\right), 0<t_{0}<1 /(4 a)$.

Conclusions A, B, C follow by an application of the author's Theorem 2, $[1944 ; 89]$, taking account of equation (4.2). Conclusion $D$ results from the fact that both integrals (4.2) are defined and continuous on the $t$-axis, $0<t$ $<1 /(4 a)$, and have equal values there.

Let us next consider the effect on the function (4.1) if $\phi(y)$ vanishes in a neighborhood of infinity. We shall need to know that this function tends to zero as $x \rightarrow+\infty$.

THEOREM 4. If

1. $F_{A}(x, t)=\int_{0}^{A}[k(x-y, t)-k(x+y, t)] \phi(y) d y$,

2. $g(x)=\operatorname{Max}_{0 \leqq t \leqq c}\left|F_{A}(x, t)\right|$ for some $c>0$. then 


$$
g(x)=O(1 / x)
$$$$
x \rightarrow \infty \text {. }
$$

For, if $x>A$, then

$$
\left|F_{A}(x, t)\right| \leqq \int_{0}^{A} k(x-A, t)|\phi(y)| d y .
$$

Using the definition of $k(x, t)$ and the trivial inequality

$$
x e^{-x^{2}}<1
$$

we obtain

$$
\left|F_{A}(x, t)\right| \leqq \frac{1}{x-A} \int_{0}^{A}|\phi(y)| d y .
$$

The right-hand side is independent of $t$, so that the conclusion of the theorem is immediate.

5. A convergence theorem. We shall now show that if $\phi(y)$ is replaced by $u(y, \delta), 0<\delta<c$, in the integral (4.1), then that integral converges provided only that $u(x, t) \in H, u(x, t) \geqq 0$ in $0<x<\infty, 0<t<c$. We prove also that its value is not greater than $u(x, t+\delta)$. In fact we shall show later that it is the equality and not the inequality that holds when $u(0, t)=0$.

THEOREM 5. If

1. $u(x, t) \in H, 0<x<\infty, 0<t<c$,

2. $u(x, t) \geqq 0,0<x<\infty, 0<t<c$,

then for $0<\delta<c, 0<x<\infty, 0<t<c-\delta$,

$$
\int_{0}^{\infty}[k(x-y, t)-k(x+y, t)] u(y, \delta) d y \leqq u(x, t+\delta) .
$$

Set

$$
F_{A}(x, t)=\int_{0}^{A}[k(x-y, t)-k(x+y, t)] u(y, \delta) d v
$$

and consider the function

$$
v(x, t)=u(x, t+\delta)-F_{A}(x, t) .
$$

By Theorem 2, $v(x, t) \in H$ in $0<t<c-\delta, 0<x<\infty$. By Conclusion $\mathrm{C}$ of Theorem 3, $v(x, t) \rightarrow 0$ as $(x, t) \rightarrow\left(x_{0}, 0+\right), 0<x_{0}<A$. By B of Theorem 3, we have as $(x, t) \rightarrow(0+, 0+)$

$$
\lim \inf v(x, t) \geqq u(0, \delta)-u(0, \delta)=0 .
$$

By virtue of the positiveness of $u(x, t)$

$$
v(x, t) \geqq-F_{A}(x, t),
$$


so that

$$
\lim \inf v(x, t) \geqq 0
$$

as $(x, t)$ approaches a point on the $t$-axis or as $(x, t)$ approaches a point $\left(x_{0}, 0+\right), x_{0}>A$.

Now to produce a contradiction make the fallacious assumption that $u\left(x_{0}, t_{0}\right)=-l<0$ at some point $0<x_{0}<\infty, 0<t_{0}<c-\delta$. By Theorem 4 we may choose $R$ so large (and $>x_{0}$ ) that $v(R, t) \geqq-l / 2,0<t<c-\delta$. Apply Theorem A to the function $v(x, t)+(l / 2)$ on the rectangle $0<x<R, 0<t<c$ $-\delta$. We conclude that $v(x, t)+(l / 2) \geqq 0$ inside that rectangle, and this gives a contradiction at $\left(x_{0}, t_{0}\right)$. Hence $v(x, t) \geqq 0$ and

$$
F_{A}(x, t) \leqq u(x, t+\delta), \quad 0<x<\infty, 0<t<c-\delta .
$$

Let $A \rightarrow \infty$. Since the integrand (5.2) is $\geqq 0$, the integral (5.1) converges, and the proof is complete.

6. A uniqueness theorem for positive functions. The theorem to be proved states in effect that if the temperature of a semi-infinite rod is never negative, is initially zero, and if the finite end of the bar is held at $0^{\circ}$, then the temperature remains at $0^{\circ}$. Although this appears physically self-evident, it should be considered in the light of such functions as (1.2).

Theorem 6. If

1. $u(x, t) \in H, 0<x<\infty, 0<t \leqq c$,

2. $u(x, t) \geqq 0,0<x<\infty, 0<t \leqq c$,

3. $\lim u(x, t)=0$ as $(x, t) \rightarrow\left(0+, t_{0}\right), 0 \leqq t_{0} \leqq c$,

4. $\lim u(x, t)=0$ as $(x, t) \rightarrow\left(x_{0}, 0+\right), 0 \leqq x_{0}<\infty$, then $u(x, t)=0$ for $0<t \leqq c, 0<x<\infty$.

Note that the function (1.2) does not satisfy conditions 3 or 4 because of its discontinuity at the origin.

If we set

$$
w(x, t)=\int_{0}^{t} u(x, y) d y,
$$

we see that $w(x, t)$ satisfies all the hypotheses of the theorem, but, by virtue of 2 , is in addition nondecreasing in $t$ and convex in $x$. If $w(x, t)$ is identically zero so too is $u(x, t)$ so that there is no loss in generality by assuming these additional properties in $u(x, t)$. Then the function $f(x)$ of Theorem 1 has the value

$$
f(x)=\operatorname{Max}_{0<t \leqq \delta} u(x, t)=u(x, \delta), \quad 0<\delta<c .
$$

Now observe that the function

$$
h(y)=k\left(1-y, t_{0}\right)-k\left(1+y, t_{0}\right)
$$


is a nonincreasing function of $y$ when $y$ is large since its derivative is negative when

$$
e^{-(1-y)^{2} / 4 t_{0}}(1-y)+e^{-(1+y)^{2} / 4 t_{0}}(1+y)<0,
$$

or when

$$
e^{-y / t_{0}}<\frac{y-1}{y+1}
$$

This inequality is clearly satisfied for large $y$.

Now by Theorem 5 we have for any $x>0$

$$
\int_{x}^{3 x} h(y) f(y) d y \leqq u\left(1, t_{0}+\delta\right) .
$$

By the convexity of $f(y)$ and the decreasing character of $h(y)$ we have for large $x$

$$
\begin{aligned}
h(3 x) \int_{x}^{3 x} f(y) d y & \leqq u\left(1, t_{0}+\delta\right), \\
2 x f(2 x) & \leqq \int_{x}^{3 x} f(y) d y .
\end{aligned}
$$

Hence for large $x$

$$
0 \leqq f(2 x) \leqq \frac{u\left(1, t_{0}+\delta\right)}{2 x h(3 x)} .
$$

From the explicit form of $h(y)$ it is evident that $1 / h(y)=O\left(e^{a y^{2}}\right), y \rightarrow+\infty$, where $a>1 /\left(4 t_{0}\right)$. From (6.2) it follows that

$$
f(x)=O\left(e^{a} x^{2}\right),
$$$$
x \rightarrow+\infty,
$$

where $a$ is any number greater than $9 /\left(16 t_{0}\right)$. We can consequently apply Theorem 1 and conclude that $u(x, t)$ is identically zero as desired.

We can now show that it is the equality that holds in (5.1) when $u(x, t)$ vanishes on the $t$-axis.

\section{Corollary 6. If in addition to the hypotheses of Theorem 5 we have}

3. $\lim u(x, t)=0$ as $(x, t) \rightarrow\left(0+, t_{0}\right), 0<t_{0}<c$, then for $0<x<\infty, 0<t<c-\delta$,

$$
\int_{0}^{\infty}[k(x-y, t)-k(x+y, t)] u(y, \delta) d y=u(x, t+\delta) .
$$

For, consider the function $v(x, t)$ which is $u(x, t+\delta)$ minus the integral (6.3). By Theorem $5, v(x, t) \geqq 0$ in $0<x<\infty, 0<t<c-\delta$. Moreover, $v(x, t) \rightarrow 0$ as $(x, t) \rightarrow\left(0+, t_{0}\right), 0 \leqq t_{0}<c-\delta$, by hypothesis 3 , and also as $(x, t) \rightarrow\left(x_{0}, 0+\right)$, 
$0 \leqq x_{0}<\infty$, by B and C of Theorem 3 . Hence by Theorem $6, v(x, t)$ is identically zero as desired.

In considering temperatures of the doubly infinite rod $(-\infty<x<\infty)$ we showed that non-negative temperature functions $u(x, t)$ must vanish identically if only $u(x, 0+)=0$ for all $x$. That is, boundary conditions could be used where the approach to the boundary (the $x$-axis) was along the normal. No corresponding result is true here, as the function (1.2) shows.

7. Representation of positive temperature functions vanishing on the $t$-axis. In this section we obtain the first of our integral representations. The conditions for representation will be seen to be both necessary and sufficient, so that the whole of the class of functions described, and no others, will be represented.

We need a simple preliminary result concerning Stieltjes integrals.

LEMMA 7. If

1. $f(x), g(x) \in C$ and $f(x), g(x)>0$,

2. $f(x) \sim a x, g(x) \sim b x, b \neq 0$,

3. $\beta(x) \in \uparrow$, $0<x \leqq 1$, $x \rightarrow 0+$, $0 \leqq x \leqq 1$,

4. $\alpha(x)=-\int_{x, g(y)}^{1} \frac{d \beta(y)}{g(,}$ $0<x \leqq 1$

then
A. $\int_{0}^{1} \frac{f(x)}{g(x)} d \beta(x)=\frac{a}{b}[\beta(0+)-\beta(0)]+\int_{0+}^{1} f(x) d \alpha(x)$,
B. $\int_{0+}^{1} x d \alpha(x)<\infty$.

By the notation of Condition 3 we mean that $\beta(x)$ is nondecreasing in the closed interval and hence bounded above by $\beta(1)$, below by $\beta(0)$. In the integral on the left of Conclusion A we understand that $f(x) / g(x)$ has been defined as $a / b$ at $x=0$ so that the quotient is continuous in $0 \leqq x \leqq 1$. For an arbitrary positive $\epsilon$ less than 1 we have

$$
\int_{0}^{1} \frac{f(x)}{g(x)} d \beta(x)=\int_{0}^{e} \frac{f(x)}{g(x)} d \beta(x)+\int_{\epsilon}^{1} f(x) d \alpha(x) .
$$

Allowing $\epsilon$ to approach $0+$, we obtain Conclusion $\mathrm{A}$, thus guaranteeing the convergence of the integral on the right of $A$. [Note that $\alpha(x)$ may $\rightarrow-\infty$ as $x \rightarrow 0+$.] Also

$$
\int_{\epsilon}^{1} x d \alpha(x)=\int_{0}^{1} \frac{x}{g(x)} d \beta(x) \leqq \int_{0+}^{1} \frac{x}{g(x)} d \beta(x)<\infty .
$$


The last integral certainly converges since $x / g(x) \rightarrow 1 / b$ as $x \rightarrow 0+$. Allowing $\epsilon$ to approach $0+$ we obtain Conclusion B.

\section{THEOREM 7. The conditions}

1. $u(x, t) \in H, \geqq 0,0<x<\infty, 0<t<c$,

2. $\lim u(x, t)=0$ as $(x, t) \rightarrow\left(0+, t_{0}\right), 0<t_{0}<c$, are necessary and sufficient that

$$
u(x, t)=p x t^{-1} k(x, t)+\int_{0+}^{\infty}[k(x-y, t)-k(x+y, t)] d \alpha(y),
$$

where $p \geqq 0, \alpha(y) \in \uparrow$ in $0<y<\infty$, the integral converges in $0<x<\infty, 0<t<c$, and

$$
\int_{0+}^{1} y d \alpha(y)<\infty .
$$

We prove first the necessity of the conditions, assuming the representation (7.1). Since the constant $p$ and the kernel of the transform (7.1) are $\geqq 0$, $0<x, 0<t$, the positiveness of $u(x, t)$ is evident. That $u(x, t) \in H$ will follow from the author's Theorem $6[1944 ; 92]$ after the integral $(7.1)$ is written as the difference of two others as in equation (4.2), provided the separate integrals converge. But if the integral (7.1) converges for $t=t_{0}<c$, then

$$
\int_{0+}^{\infty} k\left(x-y, t_{1}\right) d \alpha(y)
$$

will converge for $0<t_{1}<t_{0}$ by virtue of the inequality

$$
k\left(x-y, t_{1}\right)<k\left(x-y, t_{0}\right)-k\left(x+y, t_{0}\right) .
$$

That this holds for large $y$ may be seen by dividing the right side by the left and letting $y \rightarrow \infty$. Hence the separate integrals converge for $0<t<c, 0<x$ $<\infty$, and the necessity of Condition 1 is established. Clearly $u(x, t)$ is defined and continuous on the $t$-axis, $0<t$, and has the value zero there, so that Condition 2 is also proved.

For the sufficiency of the conditions use the function (6.1) to define

$$
\beta_{\delta}(x)=\int_{0}^{x} h(y) u(y, \delta) d y, \quad 0<\delta<c, 0<t_{0}<c-\delta .
$$

Then $\beta_{8}(x) \in \uparrow$, and by Theorem 5

$$
\beta_{8}(x) \leqq u\left(1, t_{0}+\delta\right) .
$$

Moreover, by Corollary 6

$$
u(x, t+\delta)=\int_{0}^{\infty}[k(x-y, t)-k(x+y, t)][h(y)]^{-1} d \beta_{\delta}(y) .
$$


Hence

$$
u(x, t)=\lim _{\delta \rightarrow 0+} \int_{0}^{\infty}[k(x-y, t)-k(x+y, t)][h(y)]^{-1} d \beta_{\delta}(y) .
$$

Inequality (7.3) shows that $\beta_{8}(x)$ is uniformly bounded in $x$ and $\delta$ (in a neighborhood of $\delta=0$ ), so that we may apply the Helly and Helly-Bray theorems, Widder $[1941 ; 26-32]$, in the usual way to obtain

$$
u(x, t)=\int_{0}^{\infty}[k(x-y, t)-k(x+y, t)][h(y)]^{-1} d \beta(y) .
$$

Here $\beta(y) \in \uparrow$ and $0 \leqq \beta(y) \leqq u\left(1, t_{0}\right)$ for $0 \leqq y<\infty$.

Now set

$$
\alpha(y)=-\int_{x}^{1}[h(y)]^{-1} d \beta(y) .
$$

Then $\alpha(y)$ is nondecreasing. Applying Lemma 7, we have

$$
\begin{aligned}
u(x, t) & =\frac{a}{b} \beta(0+)+\int_{0+}^{\infty}[k(x-y, t)-k(x+y, t)] d \alpha(y), \\
a & =\lim _{y \rightarrow 0+}[k(x-y, t)-k(x+y, t)] / y=x k(x, t) / t, \\
b & =\lim _{y \rightarrow 0+} h(y) / y=k\left(1, t_{0}\right) / t_{0} .
\end{aligned}
$$

If $p$ is defined as $\beta(0+) / b$, which is clearly non-negative, equation (7.4) is the desired equation (7.1). The convergence of the integral (7.2) follows from B, Lemma 7.

8. Properties of a second integral transform. We consider now the transform

(8.1) $F(x, t)=\int_{0}^{t} \frac{x}{t-y} k(x, t-y) d \alpha(y)=-.2 \int_{0}^{t} k_{x}(x, t-y) d \alpha(y)$.

We show first that $F(x, t) \in H$.

THEOREM 8. If $\alpha(y)$ is of bounded variation in $0 \leqq y \leqq c$, and if $F(x, t)$ is the function (8.1), then $F(x, t) \in H$ in $0<x<\infty, 0<t<c$.

For, $k_{x}(x, t) \in H$ in that region. The formal differentiation of the integral (8.1) needed to prove the theorem is certainly valid since only proper integrals are involved.

THEOREM 9. If $\alpha(y)$ is of bounded variation in $0 \leqq y \leqq c$ and if

$$
F(x, t)=-2 \int_{0}^{t} k_{x}(x, t-y) \alpha(y) d y,
$$


then when $(x, t) \rightarrow\left(0, t_{0}\right)$ with $0<x, 0<t \leqq t_{0}$,

$$
\lim F(x, t)=\alpha\left(t_{0}-\right), \quad 0<t_{0} \leqq c .
$$

Observe first that the theorem is true if $\alpha(y)=1$, since

$$
\lim \frac{2}{\pi^{1 / 2}} \int_{x /(4 t)^{1 / 2}}^{\infty} e^{-v^{2}} d v=1, \quad(x, t) \rightarrow\left(0, t_{0}\right), 0<t_{0} .
$$

Hence we need only show that

$$
\lim \int_{0}^{t} k_{x}(x, t-y)\left[\alpha(y)-\alpha\left(t_{0}-\right)\right] d y=0 .
$$

Given $\epsilon>0$, we determine $\delta$ so that when $t_{0}-\delta \leqq y \leqq t_{0}$

$$
\left|\alpha(y)-\alpha\left(t_{0}-\right)\right|<\epsilon,
$$

and write the integral (8.3) as the sum of two others, $I_{1}$ and $I_{2}$, corresponding respectively to the intervals of integration $\left(0, t_{0}-\delta\right),\left(t_{0}-\delta, t\right)$. Then when the point $(x, t), x>0,0<t \leqq c$, is within a distance $\delta$ of the point $\left(0, t_{0}\right)$, we have

$$
\begin{aligned}
\left|I_{2}\right| & <-\epsilon \int_{t_{0}-\delta}^{t} k_{x}(x, t-y) d y \\
& <\frac{\epsilon x}{(4 \pi)^{1 / 2}} \int_{0}^{\infty} \frac{e^{-x / 4 y}}{y^{3 / 2}} d y=\epsilon .
\end{aligned}
$$

If $M$ is an upper bound for $|\alpha(y)|$ on $0 \leqq y \leqq c$,

$$
\left|I_{1}\right| \leqq-2 M \int_{0}^{t_{0}-\delta} k_{x}(x, t-y) d y<\frac{M c x}{(4 \pi)^{1 / 2}\left(t-t_{0}+\delta\right)^{3 / 2}} .
$$

Inequalities (8.4) and (8.5) taken together establish the limit (8.3).

Corollary 9. If $\alpha(y)$ is continuous at $t_{0}, 0<t_{0} \leqq c$, then

$$
\lim F(x, t)=\alpha\left(t_{0}\right)
$$

when $(x, t) \rightarrow\left(0, t_{0}\right)$ with $0<x, 0<t \leqq c$.

For, now, $\left|\alpha(y)-\alpha\left(t_{0}\right)\right|<\epsilon$ when $\left|y-t_{0}\right|<\delta$ and inequality (8.4) holds also if $t>t_{0}$ and $(x, t)$ is within a distance $\delta$ of $\left(0, t_{0}\right)$.

Theorem 9 serves to invert the integral (8.2). If the transform is in the Stieltjes form (8.1) another inversion formula is required. It is contained in

THEOREM 10. Under the hypotheses of Theorem 8

$$
\lim _{x \rightarrow 0+} \int_{0}^{t_{0}} F(x, y) d y=\alpha\left(t_{0}-\right)-\alpha(0), \quad 0<t_{0} \leqq c .
$$


For,

$$
\begin{aligned}
\int_{0}^{t_{0}} F(x, y) d y & =-2 \int_{0}^{t_{0}} d y \int_{0}^{y} k_{x}(x, y-z) d \alpha(z) \\
& =-2 \int_{0}^{t_{0}} d \alpha(z) \int_{z}^{t_{0}} k_{x}(x, y-z) d y .
\end{aligned}
$$

The change in the order of integration is valid since the integral

$$
2 \int_{z}^{t_{0}}\left|k_{x}(x, y-z)\right| d y=\frac{x}{(4 \pi)^{1 / 2}} \int_{0}^{t_{0}-\varepsilon} \frac{e^{-x^{2} / 4 v}}{v^{3 / 2}} d v
$$

defines a continuous function of $z$ for $0 \leqq z \leqq t_{0}$. Integration by parts in (8.6) gives

$$
-2 \int_{0}^{t_{0}} k_{x}\left(x, t_{0}-z\right) \alpha(z) d z+2 \alpha(0) \int_{0}^{t_{0}} k_{x}(x, y) d y .
$$

By Theorem 9, this tends to $\alpha\left(t_{0}-\right)-\alpha(0)$ as $x \rightarrow 0+$, and the theorem is proved.

Corollary 10. A function $F(x, t)$ cannot have two essentially distinct representations in the form (8.1).

For if $\alpha_{1}(y)$ and $\alpha_{2}(y)$ were two normalized functions of bounded variation having the same transform (8.1) equal to $F(x, t)$, we should have for the difference $\alpha_{1}(y)-\alpha_{2}(y)=\alpha(y)$

$$
\int_{0}^{t} k_{x}(x, t-y) d \alpha(y)=0, \quad 0<x<\infty, 0<t<c .
$$

By Theorem 10, $\alpha_{1}(y-)=\alpha_{2}(y-)$. Since both functions are normalized, they are identical.

9. Representation of positive temperature functions vanishing on the $x$-axis. We treat next a problem like that of $\$ 7$, the boundary condition $u(0, t)=0$ being here replaced by $u(x, 0)=0$.

THEOREM 11. The conditions

1. $u(x, t) \in H, \geqq 0,0<x<\infty, 0<t<c$,

2. $\lim u(x, t)=0$ as $(x, t) \rightarrow\left(x_{0}, 0+\right), 0<x_{0}<\infty$, are necessary and sufficient that

$$
u(x, t)=-2 \int_{0}^{t} k_{x}(x, t-y) d \alpha(y),
$$

where $\alpha(y) \in \uparrow$ in $0 \leqq y<c$.

By Theorem 8 it is clear that the representation (9.1) implies Condition 1 
$\left[-k_{x}(x, y)>0\right.$ when $\left.x>0\right]$. From the elementary inequality

$$
x^{3} e^{-x^{2}}<1,
$$

$0 \leqq x<\infty$,

we obtain

$$
u(x, t) \leqq \frac{A}{x^{2}} \int_{0}^{t} d \alpha(y), \quad 0<x<\infty, 0<t<c,
$$

for a suitable constant $A$. From this inequality, Condition 2 is evident, and the necessity of the conditions is proved.

To prove that the conditions are sufficient, set

$$
\begin{array}{ll}
F(x, t)=-2 \int_{0}^{t} k_{x}(x, t-y) u(\delta, y) d y, & 0<\delta, \\
v(x, t) & =u(x+\delta, t)-F(x, t) .
\end{array}
$$

Observe first that $F(x, t)$ tends to zero as $x \rightarrow \infty$ uniformly on any interval $0 \leqq t \leqq a, a<c$. For, by $(9.2)$

$$
0 \leqq F(x, t) \leqq \frac{A}{x^{2}} \int_{0}^{a} u(\delta, y) d y, \quad 0 \leqq t \leqq a .
$$

By Theorem 9, $v(x, t) \rightarrow 0$ as $(x, t) \rightarrow\left(0+, t_{0}\right), 0<t_{0}<c$. Since

$$
0 \leqq F(x, t) \leqq \operatorname{Max}_{0 \leqq \nu \leqq t} u(\delta, y) \int_{0}^{t} \frac{x}{y} k(x, y) d y,
$$

we see that $F(x, t) \rightarrow 0$ as $(x, t) \rightarrow\left(x_{0}, 0+\right), 0<x_{0}<\infty$. For, the integral (9.4) is less than 1 and its coefficient tends to zero as $t \rightarrow 0+$ by Hypothesis 2 . We have thus shown that $v(x, t)$ approaches zero as $(x, t)$ approaches the $x$-axis or the $t$-axis. By Hypothesis 1 it can be made greater than an arbitrary negative number $-\epsilon$ on the segment $x=R, 0 \leqq t \leqq a<c$ by choosing $R$ sufficiently large. Now apply Theorem A to the function $v(x, t)+\epsilon$ on the rectangle $0 \leqq x \leqq R$, $0 \leqq t \leqq a$ and conclude that

$$
F(x, t) \leqq u(x+\delta, t), \quad 0<x<\infty, 0<t<c .
$$

Then since the function $u(x+\delta, t)-F(x, t)$ satisfies all the conditions of Theorem 6 , we see that

$$
u(x+\delta, t)=F(x, t) .
$$

Now choose an arbitrary positive number $a<c$ and set

$$
\beta_{\delta}(t)=-2 \int_{0}^{t} k_{x}(1, a-y) u(\delta, y) d y, \quad 0 \leqq t \leqq a .
$$

Then $\beta_{8}(t) \in \uparrow$ and $0 \leqq \beta_{8}(t) \leqq u(1+\delta, a)$ by $(9.5)$. By $(9.6)$ 


$$
u(x+\delta, t)=\int_{0}^{t} \frac{k_{x}(x, t-y)}{k_{x}(1, a-y)} d \beta_{\delta}(y) .
$$

Since $\beta_{\delta}(y)$ is bounded by any number larger than $u(1, a)$ uniformly in $\delta$, $0 \leqq \delta \leqq \delta_{0}$, we may apply the Helly and Helly-Bray theorems to obtain

$$
\begin{aligned}
u(x, t) & =\lim _{\delta \rightarrow 0+} \int_{0}^{t} \frac{k_{x}(x, t-y)}{k_{x}(1, a-y)} d \beta_{\delta}(y) \\
& =\int_{0}^{t} \frac{k_{x}(x, t-y)}{k_{x}(1, a-y)} d \beta(y),
\end{aligned}
$$

where

$$
\beta(y) \in \uparrow \text { in } 0 \leqq y \leqq a \text { and } 0 \leqq \beta(y) \leqq u(1, a) .
$$

If we set

$$
\alpha(t)=-\frac{1}{2} \int_{0}^{t} \frac{d \beta(y)}{k_{x}(1, a-y)}, \quad 0 \leqq t<a,
$$

equation (9.7) becomes

$$
u(x, t)=-2 \int_{0}^{t} k_{x}(x, t-y) d \alpha(y), \quad 0<x<\infty, 0<t<a .
$$

Since $k_{x}(1, a-y)$ is negative, $\alpha(t)$ is nondecreasing. Although $\alpha(t)$ appears to depend on the choice of the constant $a$, it does not really do so [both $k_{x}(1, a-y)$ and $\beta(y)$ depend on $a$ ], as one sees by use of Corollary 10 . Since the constant $a$ was arbitrary, the representation (9.8) is valid for $0<t<c$, and the proof is complete.

10. Temperature functions positive in a quadrant. We are finally in a position to attack the main problem of this paper, the representation of functions of class $H$ which are non-negative in a quadrant or in a half strip.

\section{THEOREM 12. The conditions}

1. $u(x, t) \in H, 0<x<\infty, 0<t<c$,

2. $u(x, t) \geqq 0,0<x<\infty, 0<t<c$, are necessary and sufficient that

$$
u(x, t)=\int_{0+}^{\infty}[k(x-y, t)-k(x+y, t)] d \alpha(y)-2 \int_{0}^{t} k_{x}(x, t-y) d \beta(y),
$$

the improper integral converging in $0<x<\infty, 0<t<\infty, \alpha(y) \in \uparrow$ in $0<y<\infty$, $\beta(y) \in \uparrow$ in $0 \leqq y<c$.

Note that $\alpha(y)$ may vary between $-\infty$ and $+\infty$ but that $\beta(y)$ is bounded below, $0 \leqq \beta(y)<\infty$.

The necessity of the conditions is immediate from Theorems 7 and 11. 
For the sufficiency set

$$
v_{\delta}(x, t)=\int_{0}^{\infty}[k(x-y, t)-k(x+y, t)] u(y, \delta) d y .
$$

We can no longer conclude as in Corollary 6 that

$$
v_{\delta}(x, t)=u(x, t+\delta)
$$

since Hypothesis 3 of that corollary is now missing. But by Theorem 5 we still have

$$
v_{\delta}(x, t) \leqq u(x, t+\delta), \quad 0<x<\infty, 0<t<c-\delta .
$$

That is, the function

$$
w_{\delta}(x, t)=u(x, t+\delta)-v_{\delta}(x, t)
$$

is non-negative in the region (10.1). Moreover,

$$
w_{8}(x, t) \rightarrow 0 \text { as }(x, t) \rightarrow\left(x_{0}, 0+\right)
$$

by Conclusion $\mathrm{C}$, Theorem 3. That is, $w_{8}(x, t)$ satisfies the conditions of Theorem 11 , so that by $(9.7)$

$$
w_{\delta}(x, t)=\int_{0}^{t} \frac{k_{x}(x, t-y)}{k_{x}(1, a-y)} d b_{\delta}(y), \quad 0 \leqq b_{\delta}(y) \leqq w_{\delta}(1, a) \leqq u(1, a+\delta) .
$$

Hence

$$
\begin{aligned}
u(x, t+\delta)= & \int_{0}^{\infty}[k(x-y, t)-k(x+y, t)][h(y)]^{-1} d a_{\delta}(y) \\
& +\int_{0}^{t} \frac{k_{x}(x, t-y)}{k_{x}(1, a-y)} d b_{\delta}(y),
\end{aligned}
$$

where $h(y)$ is the function (6.1) and

$$
a_{\delta}(x)=\int_{0}^{x} h(y) u(y, \delta) d y \leqq u\left(1, t_{0}+\delta\right) .
$$

The inequality holds by (10.1). We now apply the Helly and Helly-Bray theorems simultaneously to the uniformly (in $\delta$ ) bounded sets of functions $a_{8}(y)$ and $b_{\delta}(y)$ to obtain

$$
\begin{aligned}
u(x, t)= & \int_{0}^{\infty}[k(x-y, t)-k(x+y, t)][h(y)]^{-1} d a(y) \\
& +\int_{0}^{t} \frac{k_{x}(x, t-v)}{k_{x}(1, a-y)} d b(y),
\end{aligned}
$$

where $a(y)$ and $b(y)$ are bounded nondecreasing functions. 
Now proceed as in the proofs of Theorems 7 and 11, obtaining

$$
\begin{aligned}
u(x, t)=p x t^{-1} k(x, t) & +\int_{0+}^{\infty}[k(x-y, t)-k(x+y, t)] d \alpha(y) \\
& -2 \int_{0}^{t} k_{x}(x, t-y) d \beta^{*}(y)
\end{aligned}
$$

where $p \geqq 0, \alpha(y) \in \uparrow$ in $0<y<\infty, \beta^{*}(y) \in \uparrow$ in $0 \leqq y<c$. Finally we may include the first term on the right of (10.2) in the second integral if we write

$$
\begin{array}{ll}
\beta(y)=\beta^{*}(y)+p, & 0<y<c, \\
\beta(0) & =\beta^{*}(0) .
\end{array}
$$

Then (10.2) becomes the desired conclusion of the theorem.

\section{BiBLIOGRAPHY}

1. H. S. Carslaw and J. C. Jaeger, Conduction of heat in solids, Oxford, 1948.

2. P. Hartman and A. Wintner, On the solutions of the equation of heat conduction, Amer. J. Math. vol. 72 (1950) pp. 367-395.

3. A. Tychonoff, Theorèmes d'unicité pour l'equation de chaleur, Rec. Math. (Mat. Sbornik) vol. 42 (1935) pp. 199-215.

4. D. V. Widder, Positive temperatures on an infinite rod, Trans. Amer. Math. Soc. vol. 55 (1944) pp. 85-95.

HARVARD UNIVERSITY,

Cambridge, Mass. 